\title{
Влияние юстировки на плазмонную силу в субволновой щели
}

\author{
А.К. Туснин ${ }^{1,2}$, Л.Л. Фрумин ${ }^{1,2}$, О.В. Белай ${ }^{1}$ Д.А. Шапиро ${ }^{1,2, *}$ \\ ${ }^{1}$ Институт автоматики и электрометрии СО РАН, Новосибирск \\ ${ }^{2}$ Новосибирский государственныци университет, Новосибирск \\ *E-mail: shapiro@iae.nsk.su
}

DOI:10.31868/RFL2018.35-36

Исследовано влияние неточной юстировки на светоиндуцированную силу между двумя параллельными металлическими плоскостями на субволновом расстоянии. Показано, что изменение силы для непараллельных пластин имеет первый порядок по углу наклона, если пластины наклонены вдоль направления падающей волны. Ненулевой угол падения приводит к уменьшению силы из-за антисимметричной волноводной моды. В широкой щели существенной становится вторая симметричная мода, также приводящая к уменьшению силы.

Плазмонные силы $[1,2]$ остаются в центре внимания из-за их возможных применений для манипуляций частицами. Обсуждаются и другие перспективные практические приложения, такие как «тягловый луч» [3] и даже плазмонное реактивный двигатель для микроспутников [4]. В работе [5] предсказана светоиндуцированная сила между параллельными металлическими пластинами на расстоянии, много меньшем длины волны. Когда в щель падает s-волна, сила отталкивающая, а в случае р-волны - притягивающая. Светоиндуцированная сила может быть использована для манипуляции субмикронными металлическими частицами и в технике микропереключателей. Цель данной работы - исследование эффектов неточной юстировки, наклонного падения, конечной ширины щели. Поскольку в первом порядке возмущение силы представляет собой сумму всех поправок, мы вычисляем каждую отдельно. Полученные поправки важны для прецизионной юстировки экспериментальной установки.

1. Исследована сила между почти параллельными металлическими пластинами. Рассмотрен малый наклон пластин вдоль направления падения света. В случае идеально проводящих стенок найдено точное решение уравнения Гельмгольца методом разделения переменных в полярных координатах. Установлено, что поправка к силе имеет первый порядок по углу.

2. Рассмотрен случай наклона пластин в направлении, перпендикулярном падающей волне. Методом Мигдала построен ряд теории возмущений. Показано, что изменение силы имеет второй порядок по углу наклона и поэтому им можно пренебречь.

3. Изучено изменение плазмонной силы при малом отклонении падающего на щель излучения от нормали. В пределе узкой щели найдены аналитические выражения для поправок. Показано, что отклонение падающей волны от нормали приводит к возбуждению в щели первой антисимметричной моды и изменению зависимости силы от ширины щели. Возникает поправка к силе первого порядка по углу, которая уменьшает притяжение. С ростом ширины щели появляется нейтральная точка, где притяжение сменяется отталкиванием [6].

4. Первая антисимметричная мода не возбуждается при нормальном падении. Когда ширина щели много меньше длины волны, вторая симметричная мода является эванесцентной. Однако на пороге превращения моды в 
распространяющуюся волну ее роль возрастает за счет интерференции с нулевой модой, что приводит к резкому уменьшению силы вплоть до перехода от притяжения к отталкиванию. Показано, что порог в золоте отличается всего на несколько процентов от предельного случая идеального проводника [7].

\section{Литература}

[1] S. V. Perminov, V. P. Drachev et al, Opt. Express 15, 8639-8648 (2007).

[2] M. Ghorbanzadeh, S. Darbari et al, Applied Physics Letters 108, 111105 (2016).

[3] M. I. Petrov, S. V. Sukhov et al Laser \& Photonics Reviews 10, 116-122 (2016).

[4] J. N. Maser, L. Li et al, Journal of Spacecraft and Rockets 53, 998-1000 (2016).

[5] V. Nesterov, L. Frumin et al, EPL (Europhysics Letters) 94, 64002 (2011).

[6] L. L. Frumin, A. K. Tusnin at al Opt. Express 25, 31801-31809 (2017).

[7] D. Shapiro, D. Nies et al, Opt. Express 24, 15972-15977 (2016). 